\title{
Room-temperature continuous-wave operation of Ti:sapphire buried channel-waveguide lasers fabricated via proton implantation
}

\author{
C. Grivas, D. P. Shepherd, and R. W. Eason \\ Optoelectronics Research Centre, University of Southampton, Southampton SO17 1BJ, UK \\ L. Laversenne and P. Moretti \\ Laboratoire de Physico-Chimie des Matériaux Luminescents, UMR5620 CNRS, Université Claude Bernard Lyon 1 , \\ F-69622 Villeurbanne, France \\ C. N. Borca \\ Advanced Photonics Laboratory, Institute of Imaging and Applied Optics, Ecole Polytechnique Fédérale de Lausanne, \\ CH-1015 Lausanne, Switzerland \\ M. Pollnau \\ Advanced Photonics Laboratory, Institute of Imaging and Applied Optics, Ecole Polytechnique Fédérale de Lausanne, \\ CH-1015 Lausanne, Switzerland, and MESA + Research Institute, University of Twente, NL-7500 AE Enschede, \\ The Netherlands \\ Received July 3, 2006; revised August 29, 2006; accepted September 8, 2006; \\ posted September 13, 2006 (Doc. ID 72614); published November 9, 2006 \\ Fabrication and laser operation of proton-implanted Ti:sapphire buried channel waveguides is reported for \\ the first time to our knowledge. Without any postimplantation annealing of the structures, continuous laser \\ operation near $780 \mathrm{~nm}$ was demonstrated at room temperature at an absorbed pump power threshold of \\ $230 \mathrm{~mW}$. Single-transverse-mode laser emission was observed with measured beam propagation factors $M_{x}^{2}$ \\ and $M_{y}^{2}$ of 1.5 and 1.2, respectively. An output power of $12.4 \mathrm{~mW}$ for $1 \mathrm{~W}$ pump power was obtained with an \\ output coupler of $4.6 \%$ transmission at the signal wavelength. Higher output powers were measured in \\ waveguides with larger cross sections exhibiting multimode laser emission. (C) 2006 Optical Society of \\ America \\ OCIS codes: $130.3120,230.7380,140.3590,140.3570,230.7370,350.3850$.
}

Proton implantation has recently attracted interest for fabrication of waveguide lasers, because protons, compared to higher-mass ions, allow for larger penetration depths and, therefore, can lead to deeper damage profiles. They also create less damage in the guiding region, which extends the prospects of developing waveguide devices. ${ }^{1,2}$ This method has recently been proved capable of producing low-loss $(\sim 0.7 \mathrm{~dB} / \mathrm{cm})$ buried channel waveguides in undoped sapphire crystals without any postimplantation annealing. ${ }^{2}$

Ti:sapphire is a widely used laser system with broad tunability $(650-1100 \mathrm{~nm})$, which makes it suitable for the development of short pulse and broadly tunable lasers, ${ }^{3}$ with potential applications in areas as diverse as biomedical imaging, spectroscopy, sensing, and microscopy. Due to its low peak emission cross section and the short fluorescence lifetime, Ti:sapphire lasers require high pump-power densities to achieve efficient cw lasing. Development of miniature Ti:sapphire channel waveguide lasers would offer the possibility to achieve low laser thresholds as a result of the confinement of the laser and pump modes. Such lasers have been realized in planar waveguide geometry via pulsed laser deposition (PLD), ${ }^{4}$ and in channel waveguide geometry via thermal diffusion of $\mathrm{Ti}_{2} \mathrm{O}_{3}$ into sapphire, ${ }^{5}$ and a combination of PLD and photolithography-Ar+-beam milling, ${ }^{6}$ respectively. However, the indiffused channel waveguides showed low slope efficiencies, typically of the order of $\sim 0.1 \%,{ }^{5}$ while those produced by the latter method exhibited relatively high propagation losses $(\sim 1.8 \mathrm{~dB} / \mathrm{cm})$. These losses can be an unavoidable consequence of the PLD process itself and may, therefore, limit the prospects for future device developments.

Here, we report, for the first time to our knowledge, the laser operation of Ti:sapphire buried channel waveguides fabricated by proton implantation. To date, lasing action in proton-implanted crystalline waveguides has been demonstrated in garnet-based (Nd:YAG) planar structures with guidance in one direction only. ${ }^{1,7}$

Implantations were performed with high-energy protons $(0.5-1 \mathrm{MeV})$ resulting in negative refractive index changes of the order of -0.5 to $-1 \%$ for a $1 \mathrm{MeV}$ proton beam and doses between $2 \times 10^{16}$ and 4 $\times 10^{16} \mathrm{H}^{+} / \mathrm{cm}^{2}$. The Ti:sapphire crystal was uniformly doped with $0.12 \mathrm{wt}$. $\% \mathrm{Ti}_{2} \mathrm{O}_{3}$, which is rather at the high end of the range used for commercial Ti:sapphire lasers. Figure 1 shows the implantation design of a $5 \mu \mathrm{m}$ deep and $10 \mu \mathrm{m}$ wide channel waveguide. Light guidance in the vertical direction was achieved by fabricating one upper and two lower barriers inside the crystal, with proton energies of 0.5 , 0.95 , and $1 \mathrm{MeV}$ and doses of $0.5 \times 10^{16}, 1 \times 10^{16}$, and 


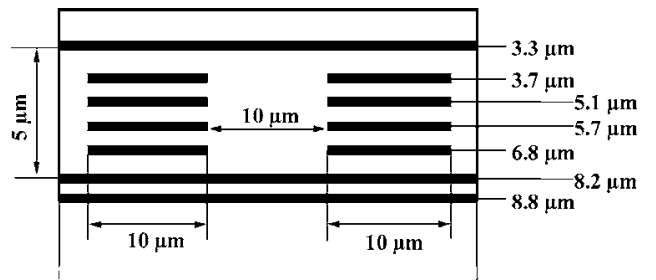

Fig. 1. Schematic (not to scale) of the implantation design of a waveguide with a cross section of $10 \mu \mathrm{m} \times 5 \mu \mathrm{m}$. Dark stripes represent the optical barriers. Their distance from the top surface of the sample is also indicated.

$1 \times 10^{16} \mathrm{H}^{+} / \mathrm{cm}^{2}$, respectively. To realize horizontal confinement, sidewalls consisting of four vertically stacked low refractive index barriers were produced via implantation through 10 or $15 \mu \mathrm{m}$ wide slits $^{8}$ with a $1 \mathrm{MeV}$ proton beam at four different angles of incidence of $65^{\circ}, 55^{\circ}, 50^{\circ}$, and $40^{\circ}$ and corresponding doses of $4 \times 10^{16}, 8 \times 10^{16}, 8 \times 10^{16}$, and 4 $\times 10^{16} \mathrm{H}^{+} / \mathrm{cm}^{2}$. By varying the angle of incidence instead of the ion energy, larger radial dispersion of the proton beam and in turn production of optical barriers with a larger width in the normal direction was achieved. As a result, several parallel $5 \mu \mathrm{m}$ deep buried channel waveguides with widths of 10,15 , and $25 \mu \mathrm{m}$ were fabricated. Prior to the optical characterization, the end faces of the sample were polished perpendicular to the channel waveguides to a high optical quality and a length of $5.1 \mathrm{~mm}$.

The fluorescence spectral emission of protonimplanted Ti:sapphire buried channel waveguides recorded at room temperature (RT) using an $\mathrm{Ar}^{+}$pump laser was very similar to that of the unimplanted bulk crystal and centered at $780 \mathrm{~nm}$ with a FWHM of $180 \mathrm{~nm}$. Nondestructive propagation loss measurements in the channel waveguides were performed via the self-pumped phase conjugation (SPPC) technique, ${ }^{9,10}$ using the $720 \mathrm{~nm}$ output from a Ti:sapphire laser and a nominally undoped $\mathrm{BaTiO}_{3}$ crystal. The experimental setup used is described in detail elsewhere. ${ }^{10}$ Losses of 1.0 and $1.3 \mathrm{~dB} / \mathrm{cm}^{2}$ at $720 \mathrm{~nm}$ were measured for the 15- and $10-\mu \mathrm{m}$-wide channels, respectively. It is suspected that narrower guides exhibit higher loss due to the stronger interaction of the modal field with their optical barriers.

Lasing experiments were performed at RT with an $\mathrm{Ar}^{+}$pump laser operating on all lines and the same optical setup for beam shaping and coupling as previously used for the loss measurements. To form the laser resonator two thin dielectric mirrors with a reflectivity of $99 \%$ and a transmission of $86 \%$ at the lasing and pump wavelength, respectively, were attached at the end faces using the surface tension of a small amount of fluorinated liquid and then by gluing their edges at the exposed parts of the faces. The output passed through a filter to block any residual pump irradiation and was then directed onto a power meter or optical spectrum analyzer (OSA) as required. CW laser action was obtained in channels with widths of 10 and $15 \mu \mathrm{m}$ at absorbed pump power thresholds of 230 and $260 \mathrm{~mW}$, respectively. Lasing was observed near $780 \mathrm{~nm}$ and the output was $\pi$ polarized regardless of the polarization state of the pump beam as a result of the larger emission cross section of the waveguide for the $\pi$ compared to the $\sigma$ polarization component. Figure 2 shows the laser spectral output as recorded by an OSA together with a laser mode profile (inset) originating from a channel with a width of $10 \mu \mathrm{m}$. The spikes in the spectrum are due to an etalon effect from the cavity mirrors and the sample, which was evident at high pump powers. The beam propagation factors $\left(M^{2}\right)$ were measured with a Coherent Modemaster beam propagation analyzer. Near-diffraction-limited laser emission was obtained from the $10-\mu \mathrm{m}$-wide channels with measured $M^{2}$ factors of $M_{x}^{2}=1.5$ and $M_{y}^{2}$ $=1.2$ for the horizontal and perpendicular direction, respectively, while the corresponding values for the 15 - $\mu \mathrm{m}$-wide channels were $M_{x}^{2}=2.5$ and $M_{y}^{2}=1.25$. By replacing the high reflective output mirror with one having a transmission of $4.6 \%$ the threshold values increased to 260 and $290 \mathrm{~mW}$ for the 10 and $15 \mu \mathrm{m}$ wide channels, respectively. Figure 3 shows the laser output characteristics as a function of the pump power for an output coupler with $4.6 \%$ transmission. Slope efficiency values of $3 \%$ and $2.2 \%$ were obtained from the 15- and 10- $\mu \mathrm{m}$-wide channels, respectively, with corresponding output powers of 17.5 and $12.4 \mathrm{~mW}$ for $1 \mathrm{~W}$ absorbed power.

To investigate the consistency of the laser performance with the measured propagation loss in the channels, an upper loss limit was derived from the experimentally obtained slope efficiency $\eta$. For this purpose we used a model that describes the effects of transverse mode profiles on $\eta$, in longitudinally pumped lasers, where the guide is a single mode at both the pump and laser wavelength. ${ }^{11}$ According to this theory the expected value for $\eta$ is given by

$$
\begin{aligned}
\eta= & \left(\frac{\lambda_{p}}{\lambda_{l}}\right)\left(\frac{-\ln \left(R_{2}\right)}{-\ln \left(R_{1} R_{2}\right)+2 l \alpha_{L}}\right) \\
& \times \eta_{q}\left(\frac{W_{l x} W_{l y}\left(2 W_{p x}^{2}+W_{l x}^{2}\right)^{1 / 2}\left(2 W_{p y}^{2}+W_{l y}^{2}\right)^{1 / 2}}{\left(W_{p x}^{2}+W_{l x}^{2}\right)\left(W_{p y}^{2}+W_{l y}^{2}\right)}\right),
\end{aligned}
$$

where $\lambda_{l}=780 \mathrm{~nm}$ is the laser and $\lambda_{p}=501 \mathrm{~nm}$ is the pump wavelength (the average of the two strongest

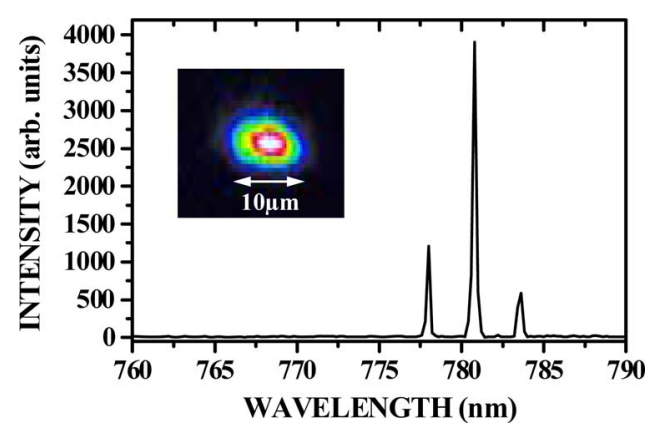

Fig. 2. (Color online) Laser emission spectrum and mode intensity profile (inset) from a Ti:sapphire buried channel waveguide with $5 \mu \mathrm{m}$ height and $10 \mu \mathrm{m}$ width. Individual lasing spikes represent etalon effects. 


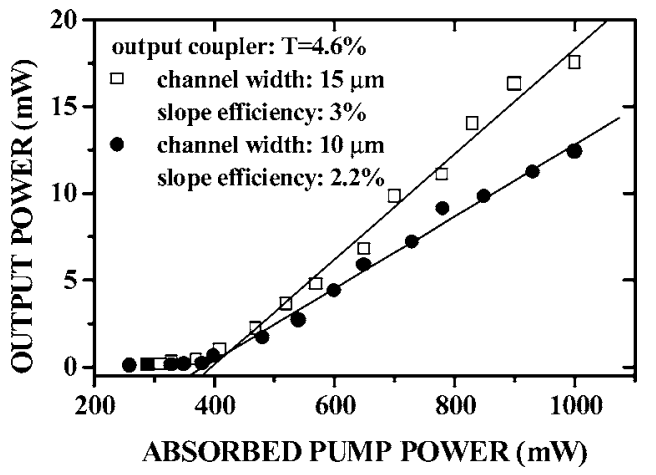

Fig. 3. Output power characteristics from two buried channel waveguides with $5 \mu \mathrm{m}$ height and widths of 15 and $10 \mu \mathrm{m}$ as a function of the absorbed pump power.

emission lines at 488 and $514 \mathrm{~nm}$ was assumed), $R_{1}$ $=0.99$ and $R_{2}=0.954$ are the reflectivities of the laser cavity input and output mirrors, $\alpha_{L}$ is the propagation attenuation coefficient, $l$ is the waveguide length, and $\eta_{q}$ is the pump quantum efficiency. Depending on the doping level and the crystal growth conditions the reported values for $\eta_{q}$ vary from 0.42 to $0.8 .^{2,12-14}$ Based on our rather high doping level and on these references we assumed a working value of 0.5 for our crystal. Low quantum efficiencies are expected for lasers operated at room temperature due to the increase in the proportion of nonradiative decay, which in turn is induced by the rise in temperature of the laser medium. The last term in Eq. (1) is the effective overlap of the pump with the lasing mode inside the waveguide, and $W_{l x}, W_{l y}, W_{p x}$, and $W_{p y}$ refer to the $1 / e^{2}$ intensity radii for the laser and pump modes in the horizontal and vertical planes, respectively. For the $10-\mu \mathrm{m}$-wide channels, exhibiting single-mode laser emission the sizes of the pump and the lasing modes were $W_{p x}=3.3 \mu \mathrm{m}$ by $W_{p y}=2.5 \mu \mathrm{m}$ and $W_{l x}=3.5 \mu \mathrm{m}$ by $W_{l y}=2.9 \mu \mathrm{m}$, respectively.

From Eq. (1) for $\eta=2.2 \%$, we obtain an upper loss limit of $<1.9 \mathrm{~dB} / \mathrm{cm}$. Considering that this value also accounts for losses due to imperfect cavity mirrors attachment and end face polishing we conclude that this estimated loss is in reasonable agreement with the value obtained by the SPPC technique $(1.3 \mathrm{~dB} / \mathrm{cm})$. Apart from scattering in the channels another reported source of loss for Ti:sapphire lasers is reabsorption of the lasing wavelength. ${ }^{2,12}$ This effect can result from the presence of $\mathrm{Ti}^{3+}-\mathrm{Ti}^{4+}$ pairs, which have increasing influence in crystals with high concentration of $\mathrm{Ti}_{2} \mathrm{O}_{3}$, as in our case, or from color centers created by the proton implantation process. A transmission spectrum recorded perpendicular to the damaged layer revealed the presence of $\mathrm{F}(E$ $=6.1 \mathrm{eV})$ and $\mathrm{F}^{+}(E=4.8 \mathrm{eV})$ color centers as previously identified in neutron-bombarded $\mathrm{Al}_{2} \mathrm{O}_{3} .{ }^{15} \mathrm{Al}-$ though such centers are localized in the damaged areas, they may act as electron traps in the guiding region and lead to creation of $\mathrm{Ti}^{4+}$ and thereby impair the laser performance by parasitic absorption. To investigate the existence of any loss component related to reabsorption, the output from a white-light source was launched into the channels and the outcoupled light was then detected by an optical multichannel analyzer. However, no absorption was observed near the lasing wavelength and therefore, we believe that the measured loss arises from scattering in the channels.

In summary, we have demonstrated what we believe is the first laser operation of buried channel waveguides fabricated via proton implantation in a Ti:sapphire crystal. Continuous-wave, singletransverse-mode laser emission has been achieved near $780 \mathrm{~nm}$ at a pump power threshold of $230 \mathrm{~mW}$. With the aim of developing a high-repetition-rate, short-pulse Ti:sapphire waveguide laser source for applications in optical coherence tomography our next immediate step is to suppress the propagation loss at a level well below $1 \mathrm{~dB} / \mathrm{cm}$. For this purpose current research is focused on the optimization of the waveguide design and implantation parameters as well as on investigating the effect of thermal annealing of the crystal on the loss. Annealing can reduce absorption via defects that may have formed during the implantation. Laser performance can also improve by incorporating directly coated reflectors at the end faces of the waveguide and adequate thermal management for the laser medium.

C. Grivas's e-mail address is chg@orc.soton.ac.uk.

\section{References}

1. M. Domenech, G. V. Vázquez, E. Márquez, R. RangelRojo, J. Rickards, and G. Trejo-Luna, Opt. Express 10, 2264 (2004).

2. L. Laversenne, P. Hoffmann, M. Pollnau, P. Moretti, and J. Mugnier, Appl. Phys. Lett. 85, 5167 (2004).

3. P. F. Moulton, J. Opt. Soc. Am. B 3, 125 (1986).

4. A. A. Anderson, R. W. Eason, L. M. B. Hickey, M. Jelinek, C. Grivas, D. S. Gill, and N. A. Vainos, Opt. Lett. 22, 1556 (1997).

5. L. M. B. Hickey, V. Apostolopoulos, R. W. Eason, J. S. Wilkinson, and A. A. Anderson, J. Opt. Soc. Am. B 21, 1452 (2004).

6. C. Grivas, D. P. Shepherd, T. C. May-Smith, R. W. Eason, and M. Pollnau, Opt. Express 13, 210 (2005).

7. M. Domenech, G. V. Vázquez, E. Flores-Romero, E. Cantelar, and G. Lifante, Appl. Phys. Lett. 86, 151108 (2005).

8. P. Moretti, M. F. Joubert, S. Tascu, B. Jacquier, M. Kaczkan, M. Malinowskii, and J. Samecki, Opt. Mater. 24, 315 (2003).

9. S. Brülisauer, D. Fluck, C. Solcia, T. Pliska, and P. Günter, Opt. Lett. 20, 1773 (1995).

10. C. Grivas, D. P. Shepherd, T. C. May-Smith, R. W. Eason, M. Pollnau, A. Crunteanu, and M. Jelinek, IEEE J. Quantum Electron. 39, 501 (2003).

11. W. A. Clarkson and D. C. Hanna, J. Mod. Opt. 36, 483 (1989).

12. P. Albers, E. Stark, and G. Huber, J. Opt. Soc. Am. B 3, 134 (1986).

13. A. Sanchez, A. J. Strauss, R. L. Aggarwal, and R. E. Fahey, IEEE J. Quantum Electron. 24, 995 (1988).

14. R. Moncorgé, G. Boulon, D. Vivien, A. M. Lejus, R. Collongues, V. Djevahirdjian, and R. Cagnard, IEEE J. Quantum Electron. 24, 1049 (1988).

15. K. H. Lee and J. H. Crawford, Jr., Phys. Rev. B 15, 4065 (1977). 